\title{
Comparação entre Três Escores Embrionários como Fator Prognóstico do Sucesso em Fertilização in vitro
}

\author{
Comparison between Three Embryo Scoring Systems as Predictive \\ of the Outcome of in vitro Fertilization \\ Marcos Dias de Moura, Vinícius Alves de Oliveira \\ Maria Matheus de Sala, Rui Alberto Ferriani, Rosana Maria dos Reis
}

\begin{abstract}
RESUMO
Objetivo: avaliar três sistemas de escore embrionário para embriões de $3^{\circ}$ dia e correlacionálos com resultados positivos da técnica de fertilização in vitro.

Método: estudo retrospectivo desenvolvido pelo programa de fertilização in vitro do Hospital das Clinicas da Faculdade de Medicina de Ribeirão Preto - USP. Foram incluidas 137 pacientes submetidas a fertilização in vitro e transferência de 439 embriões. Os principais resultados avaliados foram taxa de gravidez e taxa de implantação.

Resultados: nos três métodos observou-se diferença significativa no escore embrionário entre o grupo de grávidas $(n=53)$ e não grávidas $(n=84)(p<0,0001)$. No escore 1 , avaliando-se apenas o número de células, observou-se maior taxa de gravidez (70\%) e taxa de implantação (42\%) nas transferências de embriões com média dos blastômeros acima de 8. O escore 2, baseado num escore total de quatro pontos (clivagem, número de blastômeros, fragmentação e simetria), mostrou aumento nas taxas de gravidez (52,8\%) e implantação (31\%) nos escores acima de 2. No escore 3, baseado no número de células e no grau morfológico, também as taxas de gravidez e de implantação elevaram-se de acordo com o aumento do escore médio dos embriões transferidos.

Conclusão: Os três sistemas de escore avaliados em embriões de $3^{\circ}$ dia, correlacionaram-se positivamente com taxa de gravidez e taxa de implantação.
\end{abstract}

PALAVRAS-CHAVE: Escore embrionário. Fertilização in vitro. Taxa de gravidez. Taxa de implantação.

\section{Introdução}

Desde o início da fertilização in vitro (FIV), inúmeros trabalhos têm procurado identificar bons indicadores de resultados positivos da técnica, sendo a qualidade embrionária um dos fatores mais importantes descritos nestes estudos ${ }^{1-3}$. A qualidade dos embriões é um dos fatores que pode influenciar a taxa de gravidez, sendo considerado

Departamento de Ginecologia e Obstetrícia da Faculdade de Medicina de Ribeirão Preto da Universidade de São Paulo Correspondência:

Marcos Dias de Moura

Departamento de Ginecologia e Obstetrícia

Faculdade de Medicina de Ribeirão Preto - USP

Av. dos Bandeirantes, 3900

14049-900 Ribeirão Preto-SP

e-mail: mddmoura@fmrp.usp.br mau prognóstico a presença de fragmentos anucleados, presença de blastômeros irregulares e o baixo número de blastômeros ${ }^{4}$. Vários sistemas de classificação foram desenvolvidos para graduar a qualidade dos embriões ${ }^{5-7}$. Sabe-se que a taxa de gravidez aumenta com o número de embriões transferidos $^{8-10}$ e a taxa de implantação correlacionase positivamente com a boa morfologia e o estágio de clivagem dos embriões ${ }^{5,711-13}$.

Alguns autores propuseram sistemas de escore embrionário específicos para embrião de dois dias. As três principais características incluídas nestes sistemas de classificação embrionária foram o número de células, o tamanho e a forma dos blastômeros e o grau de fragmentação ${ }^{2,5,7,11,12,14}$. O escore morfológico médio ${ }^{2}$ e o escore embrionário cumulativo ${ }^{11,15}$ incluem também o número de embriões transferidos ao escore fi- 
nal. Giorgetti et al. ${ }^{16}$, observando a influência de quatro parâmetros embrionários na taxa de gravidez após transferência de 957 embriões únicos, criaram um escore embrionário de quatro pontos. Posteriormente, Terriou et al. ${ }^{4}$ avaliaram a influência da qualidade dos embriões na taxa de gravidez, por meio de novo escore embrionário cumulativo, obtido pela soma do escore embrionário individual, de todos os embriões transferidos, dividido pelo número desses embriões, originando um escore médio de embriões transferidos após FIV.

Poucos sistemas de escore específicos para embrião de 3 dias têm sido descritos atualmente. Desai et al. ${ }^{17}$ estabelecendo a relação entre clivagem embrionária, parâmetros morfolológicos específicos do $3^{\circ}$ dia (padrão de fragmentação, granulações citoplasmáticas, compactação, simetria, expansão e ausência de vacúolos dos blastômeros) e resultados de gestação, originaram um escore de qualidade para embrião de $3^{\circ}$ dia (D3EQ).

O objetivo deste estudo foi avaliar três sistemas de escore empregados para embriões de $2^{\circ}$ dia aplicados a embriões de $3^{\circ}$ dia, previamente classificados quanto à morfologia, procurando correlacionar com taxa de gravidez e taxa de implantação.

\section{Pacientes e Métodos}

Avaliou-se um total de 439 embriões que foram obtidos de 137 pacientes seguidas no programa de fertilização in vitro do Hospital de Clínicas da Faculdade de Medicina de Ribeirão Preto - USP, no período de julho de 1999 a julho de 2001.

Todas as pacientes foram submetidas à indução de ovulação com gonadotrofinas exógenas, associadas a protocolo longo de supressão hipofisária com agonistas do hormônio liberador das gonadotrofinas (GnRH-a). O crescimento folicular foi monitorizado por ultra-sonografias seriadas e a ovulação foi induzida com gonadotrofina coriônica humana (hCG) na dosagem de 10.000 UI, quando dois ou mais folículos atingissem diâmetro superior a 18 milimetros.

A captação dos oócitos foi realizada 36 horas após a administração do hCG e a FIV convencional foi realizada conforme protocolo do serviço. A fertilização dos oócitos foi confirmada após 18 a 20 horas com a visualização de dois pronúcleos. Os embriões permaneceram em cultura até 72 horas após a captação e foram, então, classificados quanto à morfologia. A transferência foi realizada no $3^{\circ}$ dia.

As pacientes receberam suplementação de fase lútea e o diagnóstico de gravidez foi obtido pela dosagem sérica de $\beta$ hCG 14 dias após a transferência dos embriões. Do total de 137 pacientes estudadas, $53(38,6 \%)$ engravidaram após a realização do procedimento de FIV.

Os embriões foram previamente classificados no dia da transferência (D3), quanto à morfologia ${ }^{14}$, da seguinte forma: Tipo 1.0 - Blastômeros regulares, sem fragmentação; Tipo 2.0 - Blastômeros irregulares, sem fragmentação; Tipo 2.1 Blastômeros regulares ou irregulares, $(<10 \%$ de fragmentação); Tipo 2.2 - Blastômeros regulares ou irregulares, ( $\geq 10 \%$ e $<20 \%$ de fragmentação); Tipo 3.0 - Blastômeros regulares ou irregulares, $(\geq 20 \%$ e $<50 \%$ de fragmentação) e Tipo 4.0 - Blastômeros regulares ou irregulares, ( $\geq 50 \%$ de fragmentação).

\section{Padronização dos escores embrionários}

Para obtenção do escore 1 avaliou-se apenas o número de blastômeros dos embriões transferidos. Por exemplo, embriões com uma célula tiveram escore igual a 1 , aqueles com duas células tiveram escore igual a 2 e assim sucessivamente.

O escore 2 foi obtido adaptando-se o escore embrionário de 4 pontos ${ }^{16}$ para embrião de $3^{\circ}$ dia (Tabela 1), no qual todos os embriões clivados receberam 1 ponto, e 1 ponto adicional foi acrescentado para cada uma das seguintes características: estágio de 8 ou mais blastômeros, ausência de fragmentação ou fragmentação envolvendo menos de $20 \%$ da superfície do embrião (tipo 1.0, 2.0, 2.1, 2.2) e ausência de irregularidade no tamanho ou forma do blastômero (tipo 1.0). Um escore ideal de 4 pontos foi obtido com embriões apresentando 8 ou mais células, sem fragmentação $(<20 \%$ de fragmentação) e sem irregularidade dos blastômeros.

Tabela 1 - Cálculo do escore embrionário de 4 pontos modificado para embrião de $3^{\circ}$ dia (escore 2).

\begin{tabular}{lc}
\hline & Pontos \\
\hline Embriões clivados & 1 \\
Estágio de divisão & \\
$<8$ células & 0 \\
$>8$ células & 1 \\
Fragmentos anucleados & \\
Presença & 0 \\
Ausência & 1 \\
Células irregulares & \\
Presença & 0 \\
Ausência & 1 \\
\hline
\end{tabular}

O escore 3 foi calculado a partir do escore embrionário cumulativo ${ }^{11}$, pelo qual os embriões eram classificados de acordo com o grau morfológico: grau I (tipo 4.0), grau II (tipo 3.0), grau III 
(tipo 2.2 e 2.1) e grau IV (tipo 2.0 e 1.0). O grau morfológico que avalia simetria e grau de fragmentação foi multiplicado pelo número de blastômeros, produzindo um escore para cada embrião.

\section{Métodos}

Cada embrião foi avaliado isoladamente e valores relativos a cada um dos escores considerados foram atribuídos. Visando avaliar o desempenho de cada um dos três sistemas de escore embrionário, foram comparados os valores obtidos com cada um deles nos grupos de pacientes grávidas $(n=53)$ e não-grávidas $(n=84)$. Para obtenção de resultados mais acurados, essa avaliação foi realizada de duas formas: 1) considerando-se o escore médio de todos os embriões transferidos por paciente, e 2) considerando-se o escore médio dos dois embriões de melhor qualidade por paciente.

Optou-se por avaliar cada escore também pela média dos dois melhores embriões transferidos, por acreditar-se que isto pudesse refletir melhor o potencial de gravidez em cada ciclo, uma vez que o escore total não deveria ser diluído pela presença de um $3^{\circ}$ ou $4^{\circ}$ embrião de pior qualidade.

Posteriormente, procurou-se estabelecer correlação entre os três sistemas de avaliação embrionária empregados. Para tal, os valores atribuídos para cada um dos escores foram comparados entre si, considerando-se o total de embriões estudados.

Visando correlacionar os resultados obtidos para cada um dos sistemas de escore com as taxas de gravidez e implantação, foi empregado escore médio de todos os embriões transferidos por paciente.

\section{Análise estatistica}

Para a análise estatística utilizou-se o software GraphPad Prism version 3.00 for Windows
(GraphPad Software, San Diego, CA, USA). Os valores amostrais apresentaram distribuição não paramétrica, de acordo com o teste de Kolmogorov-Smirnov. Para a comparação entre os escores observados nos grupos de grávidas e nãográvidas, foi empregado o teste de Mann-Whitney e as correlações entre os três escores embrionários foram testadas por meio do teste de correlação de Spearman. Nivel de significância de 5\% foi adotado em todos os procedimentos estatísticos. O presente trabalho foi aprovado pelo Comitê de Ética e Pesquisa do Hospital das Clínicas da Faculdade de Medicina de Ribeirão Preto da Universidade de São Paulo.

\section{Resultados}

A média de idade no grupo de pacientes grávidas e não-grávidas foi de 32,3 anos $( \pm 4,3)$ e 32,8 anos $( \pm 4,7)$, respectivamente. Das diversas indicações para o tratamento da infertilidade nessas pacientes, o fator tubário $(18,2 \%)$, a endometriose $(22,6 \%)$ e o fator masculino $(37,9 \%)$ foram responsáveis pela maioria dos casos, mas sem diferença estatística entre os dois grupos.

A maioria das pacientes recebeu $3(36,5 \%)$ ou $4(32,8 \%)$ embriões. A taxa de gravidez clínica foi de $40 \%$ com 3 embriões transferidos e $48 \%$ com 4 embriões. Não houve diferença estatisticamente significativa na distribuição da idade ou no número de embriões transferidos entre o grupo de pacientes grávidas e não grávidas.

A Tabela 2 mostra a distribuição da idade e o número de embriões transferidos, correlacionando estas variáveis com taxa de gravidez e taxa de implantação. Observou-se que, com o aumento da idade, mais embriões foram transferidos, porém houve diminuição na taxa de gravidez e na taxa de implantação.

Tabela 2 - Relação entre idade, número de embriões transferidos e resultados de gravidez (em \%).

\begin{tabular}{ccccc}
\hline $\begin{array}{c}\text { Idade } \\
\text { (anos) }\end{array}$ & $\begin{array}{c}\text { Transferências } \\
(\mathbf{n})\end{array}$ & $\begin{array}{c}\text { Média do número de } \\
\text { embriões transferidos } \\
(\mathbf{\pm D P})\end{array}$ & $\begin{array}{c}\text { Taxa gravidez } \\
\text { clínica }(\%)\end{array}$ & $\begin{array}{c}\text { Taxa implantação } \\
(\%)\end{array}$ \\
\hline$<36$ & 97 & $3,1 \pm 1.0$ & 69,7 & 25,1 \\
$36-38$ & 28 & $3,2 \pm 1.1$ & 20,7 & 12,0 \\
$>38$ & 12 & $3,5 \pm 1.5$ & 9,5 & 9,5 \\
\hline
\end{tabular}

Todos os embriões transferidos foram avaliados pelos 3 escores. Para qualquer dos escores empregados foi encontrada diferença estatisticamente significativa entre o grupo de grávidas e não-grávidas $(\mathrm{p}<0,0001)$. Considerando-se o escore médio de todos os embriões transferidos por paciente, as medianas $\left(1^{\circ} \mathrm{e} 3^{\circ}\right.$ quartis) dos valores obtidos para o escore 1 foram de $7,5(5,9-8)$ no gru- 
po de grávidas e 6 (5-7), no grupo de não-grávidas, como se pode observar na Tabela 3. Diferença significativa foi também observada ao se considerar o escore médio dos dois melhores embriões $(\mathrm{p}<0,0001)$.

Para o escore 2, os valores observados nos grupos de pacientes grávidas e não grávidas foram de 3 (com variação de 2,3 a 3,7) e 2 (com variação de 1,5 a 3), respectivamente, com diferença estatisticamente significativa (Tabela 3). Em relação ao escore 3, também foram observados maiores escores embrionários no grupo de pacientes grávidas (Tabela 3 ).

Tabela 3 - Valores dos 3 escores embrionários de todos os embriões transferidos e resultado obtido quanto a gravidez clínica.

\begin{tabular}{lcc}
\hline & $\begin{array}{c}\text { Grávidas } \\
(\mathbf{n}=\mathbf{1 8 1})\end{array}$ & $\begin{array}{c}\text { Não-grávidas } \\
(\mathbf{n}=\mathbf{2 5 8})\end{array}$ \\
\hline Escore 1 & $7,5^{*}$ & 6 \\
& $(5,9-8)$ & $(5-7)$ \\
Escore 2 & $3^{*}$ & 2 \\
& $(2,3-3,7)$ & $(1,5-3)$ \\
Escore 3 & $26,6^{*}$ & 20,7 \\
& $(21,7-32)$ & $(16,2-25)$ \\
\hline
\end{tabular}

Resultados expressos como mediana ( $1^{\circ}$ e $3^{\circ}$ quartis).

${ }^{*} p<0,0001$ comparado com não-grávidas.

De forma semelhante ao observado com o escore 1, a mesma diferença entre os grupos de grávidas e não-grávidas foi evidenciada ao se considerar o escore médio dos dois embriões de melhor qualidade com o uso dos escores 2 e 3 (Figura 1).

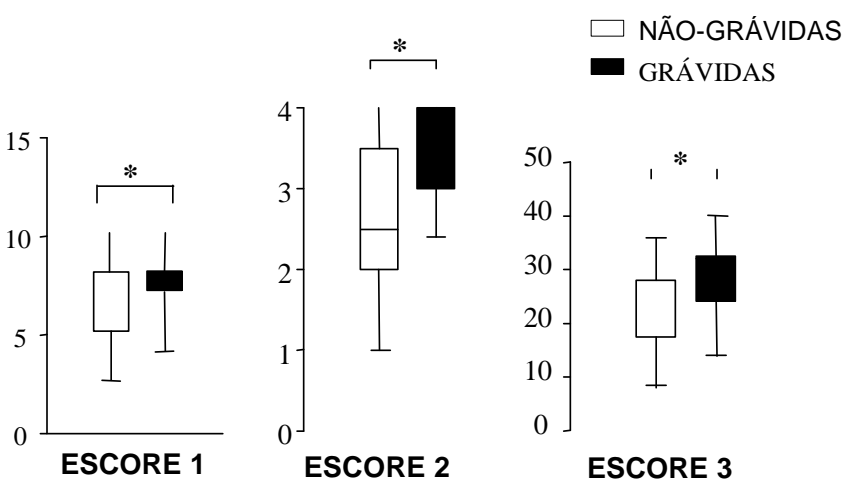

Figura 1 - Valores dos três escores embrionários pela média dos dois melhores embriões transferidos no dia 3 , em pacientes grávidas e não grávidas. Resultados expressos em mediana e $1^{\circ}$ e $3^{\circ}$ quartis. ${ }^{*}=$ teste de Mann-Whitney $(p<0,0001)$.

Foi observada correlação positiva significativa entre os três sistemas de escore embrionário, tanto no grupo de grávidas como no grupo de não-grávidas.

A relação entre a média dos embriões transferidos e a média dos dois melhores embriões foi analisada em relação à taxa de gravidez e à taxa de implantação.

Analisando-se o impacto do número de células, com o uso do escore 1 , observou-se maior taxa de gravidez clínica $(70 \%)$ e taxa de implantação $(42 \%)$ nas transferências de embriões com maior número de blastômeros ( $\geq 8$ células) (Figura 2). Utilizando-se o escore 2 , não foi observada gravidez nas transferências de embriões com média de escore menor que 2 . Obteve-se elevada taxa de gravidez $(52,8 \%)$ e taxa de implantação $(31 \%)$ nas transferências com escore entre 3-4 (Figura 2). Com relação ao escore 3 , a taxa de gravidez foi de $75 \%$ e a taxa de implantação de $42,8 \%$ no grupo de pacientes que apresentaram escore médio maior que 30 (Figura 2).

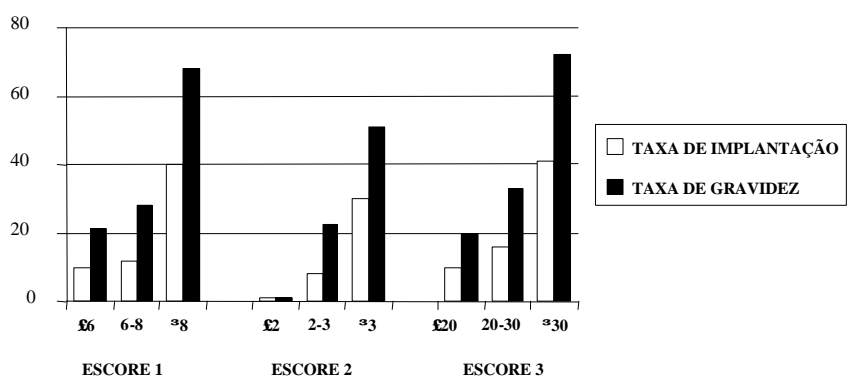

Figura 2 - Relação entre a média dos escores dos embriões transferidos baseado apenas no número de blastômeros (escore 1), estágio de clivagem, número de células, grau de fragmentação e simetria (escore 2) e número de células e grau morfológico (escore 3 ) com os índices de gravidez e taxas de implantação.

\section{Discussão}

O aumento da idade é, sabidamente, reconhecido como causa de declínio na vida reprodutiva da mulher ${ }^{18-20}$ e quando se empregam técnicas de FIV relaciona-se com a diminuição nas taxas de gravidez e taxas de implantação, principalmente após os 38 anos de idade ${ }^{4,16,17,20}$, como pode ser observado em nossos resultados.

A finalidade deste estudo foi avaliar e testar a eficácia e funcionabilidade de três métodos diferentes de escore embrionário, utilizando-se parâmetros tradicionais de sistemas de escore de embrião de $2^{\circ}$ dia, adaptados para avaliar embriões de $3^{\circ}$ dia. Devido ao fato de os embriões desse estudo terem sido previamente classificados de acordo com a classificação morfológica preestabelecida, optamos por testar métodos que avaliassem apenas critérios relacionados à clivagem, ao número de células, à simetria e à fragmentação dos blastômeros. Embora vários trabalhos demonstrassem a importância destes parâmetros embrionários na taxa de gravide $z^{5,6,12,13,21,22}$, poucos estudos levaram em conta todos esses parâmetros na classificação embrionária ${ }^{7,11}$. 
Com base em alguns estudos ${ }^{4,5,7,11,16,17}$, adaptamos três sistemas de escore de embrião de $2^{\circ}$ dia para classificar os embriões transferidos no $3^{\circ}$ dia. O número de células pareceu alterar as taxas de gravidez e implantação. Nossos achados foram compativeis com outros estudos que consideraram o número de células forte indicador de vitalidade embrionária e de resultados de gestação, tendose observado que menor estágio de clivagem embrionária influencia de maneira negativa a taxa de gravide $^{6,14,16,17,23}$.

A fragmentação tem sido freqüentemente incluída nos sistemas de escore para auxiliar na classificação embrionária ${ }^{2} \mathrm{e}$, conforme observado em outro estudo ${ }^{16}$, a taxa de gravidez aumenta quando os embriões mostram menos de $20 \%$ de fragmentação. A perda de proteínas reguladoras durante a fragmentação dos blastômeros pode ser o mecanismo pelo qual o desenvolvimento do embrião é afetado ${ }^{24}$.

Alikani et al. ${ }^{25}$, avaliando os efeitos da fragmentação dos embriões de $3^{\circ}$ dia, associaram o grau de fragmentação com a presença de alterações cromossômicas. Mais tarde, outro estudo considerou, para os sistemas de escore embrionário, o tamanho dos blastômeros como critério de maior importância que a fragmentação, observando-se que embriões clivados irregularmente tinham o grau de aneuploidias e a taxa de multinucleação maiores que aqueles com blastômeros regulares ${ }^{26}$.

Giorgetti et al. ${ }^{16}$, estudando a transferência de 957 embriões únicos de $2^{\circ}$ dia, demonstraram que a clivagem, o número de blastômeros, a presença de blastômeros irregulares e de fragmentos anucleados influenciaram os resultados de FIV e a transferência de embriões. Quanto ao número de blastômeros, sabe-se que divisão apropriada para embriões transferidos no $3^{\circ}$ dia deve resultar em um mínimo de oito células ${ }^{17}$. Em nosso estudo, observamos que com o escore 2 , a taxa de gravidez apresentou comportamento semelhante ao de ou$\operatorname{tros}^{4,16}$, nos quais se verificou que, quanto maior o escore, maiores as taxas de gravidez. Ao avaliarmos a média do escore 3 dos embriões transferidos, observamos, também, que a taxa de gravidez e a taxa de implantação aumentaram com a elevação do escore em cada transferência, o que está de acordo com os resultados obtidos por Steer et al. ${ }^{11}$.

Sabe-se que um dos problemas associados com o cálculo do escore médio de embriões transferidos é que, numa transferência mista envolvendo embriões de boa e má qualidade, o escore total pode ser mais baixo, e não necessariamente refletir o verdadeiro potencial de gestação ${ }^{17}$. Por isso, nós optamos por comparar, também, os três sistemas de escore com a média dos dois melhores embriões transferidos. Observamos que a avaliação dos dois embriões melhores classificados foi suficiente para predizer resultados de gravidez. É importante ressaltar que, como a maioria das pacientes receberam três ou quatro embriões, ao fazermos a média dos dois melhores embriões, selecionamos metade ou um terço dos embriões transferidos.

A incorporação de características específicas para embrião de $3^{\circ}$ dia a outros parâmetros de escore embrionário tradicionais (número de blastômeros, padrão de fragmentação) pode ser útil na classificação e seleção de embriões para transferência ${ }^{17}$. Estudo avaliando atributos morfológicos dos embriões de três dias observou que o aumento na granularidade citoplasmática e o aumento dos blastômeros elevaram a taxa de gestação ${ }^{27}$. Algumas características como a boa expansão do blastômero, que chega a tocar a zona pelúcida com reduzido espaço perivitelínico, o citoplasma livre de vacúolos e sinais de compactação foram selecionadas como parâmetros morfológicos importantes na classificação embrionária do $3^{\circ}$ dia $^{18}$.

Certamente, a maior limitação deste estudo foi a de aplicar os três sistemas de escore tradicionais em embriões já previamente classificados e selecionados para transferência, com informações restritas quanto à morfologia. Entretanto, pode-se concluir que os 3 sistemas de escore adaptados para classificar embriões de $3^{\circ}$ dia foram indicadores de prognósticos do resultado de gestação.

Acreditamos que um estudo prospectivo permitirá avaliar melhor os parâmetros específicos dos embriões de 3 dias e a relação desses parâmetros com o potencial de gravidez, permitindose utilizar sistema de escore específico para o $3^{\circ}$ dia que permita escolher os melhores embriões para transferência, predizer resultados de FIV e da transferência de embriões e reduzir o número de gestações múltiplas.

\section{ABSTRACT}

Purpose: to evaluate three embryo scoring systems specific for 3-day embryos and to correlate them with positive in vitro fertilization outcome.

Method: retrospective study of the In Vitro Fertilization Program of the University Hospital, Faculty of Medicine of Ribeirão Preto, University of São Paulo. A total of 137 patients submitted to the transfer of 439 embryos were evaluated. The main outcomes measured were pregnancy and implantation rates.

Results: a significant difference in the three scoring systems was observed between pregnant $(n=53)$ and non-pregnant $(n=84)$ patients $(p<0.0001)$. In the first embryo scoring system, in which cell number alone was used, higher pregnancy $(70 \%)$ and implantation rates $(42 \%)$ were 
observed when embryos with a mean blastomere number higher than 8 were transferred. Scoring system 2, based on a total four-point score (cleavage stage, blastomere number, fragmentation and symmetry), showed increased pregnancy (52.8\%) and implantation rates (31\%) for scores above 2 . Scoring system 3, based on cell number and morphological criteria, also showed higher pregnancy and implantation rates with increasing average scores of the transferred embryos.

Conclusion: the three scoring systems assessed in 3-day embryos were positively correlated with pregnancy and implantation rates.

KEYWORDS: Embryo score. In vitrofertilization. Pregnancy rate. Implantation rate.

\section{Referências}

1. Saith R, Sargent I. Embryo selection for transfer in human IVF. Assist Reprod Rev 1995; 5:145-54.

2. Roseboom TJ, Vermeiden JP, Schoute E, Lens JW, Schats $R$. The probability of pregnancy after embryo transfer is affected by the age of the patient, cause of infertility, number of embryos transferred and the average morphology score, as revealed by multiple logistic regression analysis. Hum Reprod 1995; 10:3035-41.

3. Saith RR, Srinivasan A, Michie D, Sargent IL. Relationships between the developmental potential of human in-vitro fertilization embryos and features describing the embryo, oocyte and follicle. Hum Reprod Update 1998; 4:121-34.

4. Terriou P, Sapin C, Giorgetti C, Hans E, Spach JL, Roulier R. Embryo score is a better predictor of pregnancy than the number of transferred embryos or female age. Fertil Steril 2001; 75:525-31.

5. Cummins JM, Breen TM, Harrison KL, Shaw JM, Wilson LM, Hennesey JF. A formula for scoring human embryo growth rates in in vitro fertilization: its value in predicting pregnancy and in comparison with visual estimates of embryo quality. J In Vitro Fert Embryo Transf 1986; 3:284-95.

6. Claman P, Armant DR, Seibel MM, Wang TA, Oskowitz SP, Taymor ML. The impact of embryo quality on implantation and the establishment of viable pregnancies. J In Vitro Fert Embryo Transf 1987; 4:218-22.

7. Puissant F, Van Rysselberge M, Barlow P, Deweze $\mathrm{J}$, Leroy F. Embryo scoring as a prognostic tool in IVF treatment. Hum Reprod 1987; 8:705-8.

8. Edwards RG, Steptoe PC. Current status of invitro fertilization and implantation of human embryos. Lancet 1983; 2:1265-9.

9. Kerin JF, Warnes GM, Quinn PJ, et al. Incidence of multiple pregnancy after in-vitro fertilization and embryo transfer. Lancet 1983; 2:537-40.
10.Tan SL, Steer C, Royston P, Rizk P, Mason BA, Campbell $S$. Conception rates and in-vitro fertilization. Lancet 1990; 335:299.

11.Steer CV, Mills CL, Tan SL, Campbell S, Edwards RG. The cumulative embryo score: a predictive embryo scoring technique to select the optimal number of embryos to transfer in an in-vitro fertilization and embryo transfer programme. Hum Reprod 1992; 7:117-9.

12.Staessen C, Camus M, Bollen N, Devroey P, Van Steirteghem AC. The relationship between embryo quality and the occurence of multiple pregnancies. Fertil Steril 1992; 57:626-30.

13.Shulman A, Ben-Nun I, Ghetler Y, Kaneti H, Shilon M, Beyth Y. Relationship between embryo morphology and implantation rate after in vitro fertilization treatment in conception cycles. Fertil Steril 1993; 60:123-6.

14.Ziebe S, Petersen K, Lindenberg S, Andersen AG, Gabrielsen A, Andersen AN. Embryo morphology or cleavage stage: how to select the best embryos for transfer after in-vitro fertilization. Hum Reprod 1997; 12:1545-9.

15.Visser DS, Fourie FR. The applicability of the cumulative embryo score system for embryo selection and quality control in an in-vitro fertilization/embryo transfer program. Hum Reprod 1993; 8:1719-22.

16. Giorgetti C, Terriou P, Auquier P, et al. Embryo score to predict implantation after in-vitro fertilization: based on 957 single embryo transfers. Hum Reprod 1995; 10:2427-31.

17.Desai NN, Goldstein J, Rowland DY, Goldfarb JM. Morphological evaluation of human embryos and derivation of an embryo quality scoring system specific for day 3 embryos: a preliminary study. Hum Reprod 2000; 15:2190-6.

18.Dew JE, Don RA, Hughes GJ, Johnson TC, Steigrad SJ. The influence of advanced age on the outcome of assisted reproduction. J Assist Reprod Genet $1998 ; 15: 210-4$.

19.Keefe DL. Reproductive aging is an evolutionarily programmed strategy that no longer provides adaptive value. Fertil Steril 1998; 70:204-6.

20.Ziebe S, Loft A, Petersen JH, et al. Embryo quality and developmental potential is compromised by age. Acta Obstet Gynecol Scand 2001; 80:169-74.

21.Erenus M, Zouves C, Rajamahendran P, Leung S, Fluker M, Gomel V. The effect of embryo quality on subsequent pregnancy rates after in vitro fertilization. Fertil Steril 1991; 56:707-10.

22.Monks NJ, Turner K, Hooper MA, Kumar A, Verma $\mathrm{S}$, Lenton EA. Development of embryos from natural cycle in-vitro fertilization: impact of medium type and female infertility factors. Hum Reprod 1993; 8:266-71. 
23.Lewin A, Schenker JG, Safran A, et al. Embryo growth rate in vitro as an indicator of embryo quality in IVF cycles. J Assist Reprod Genet 1994; 11:500-3.

24.Antczak M, Van Blerkom J. Temporal and spatial aspects of fragmentation in early human embryos: possible effects on developmental competence and association with the differential elimination of regulatory proteins from polarized domains. Hum Reprod 1999; 14:429-47.

25.Alikani M, Cohen J, Tomkin G, Garrisi GJ, Mack C, Scott RT. Human embryo fragmentation in vitro and its implications for pregnancy and implantation. Fertil Steril 1999; 71:836-42.
26. Hardarson T, Hanson C, Sjögren A, Lundin K. Human embryos with unevenly sized blastomeres have lower pregnancy and implantation rates: indications for aneuploidy and multinucleation. Hum Reprod $2001 ; 16: 313-8$.

27.Wiemer KE, Garrisi J, Steuerwald N, et al. Beneficial aspects of co-culture with assisted hatching when applied to multiple-failure in-vitro fertilization patients. Hum Reprod 1996; 11:2429-33.

Recebido em: 24/3/2003 Aceito após modificações em: 25/4/2003

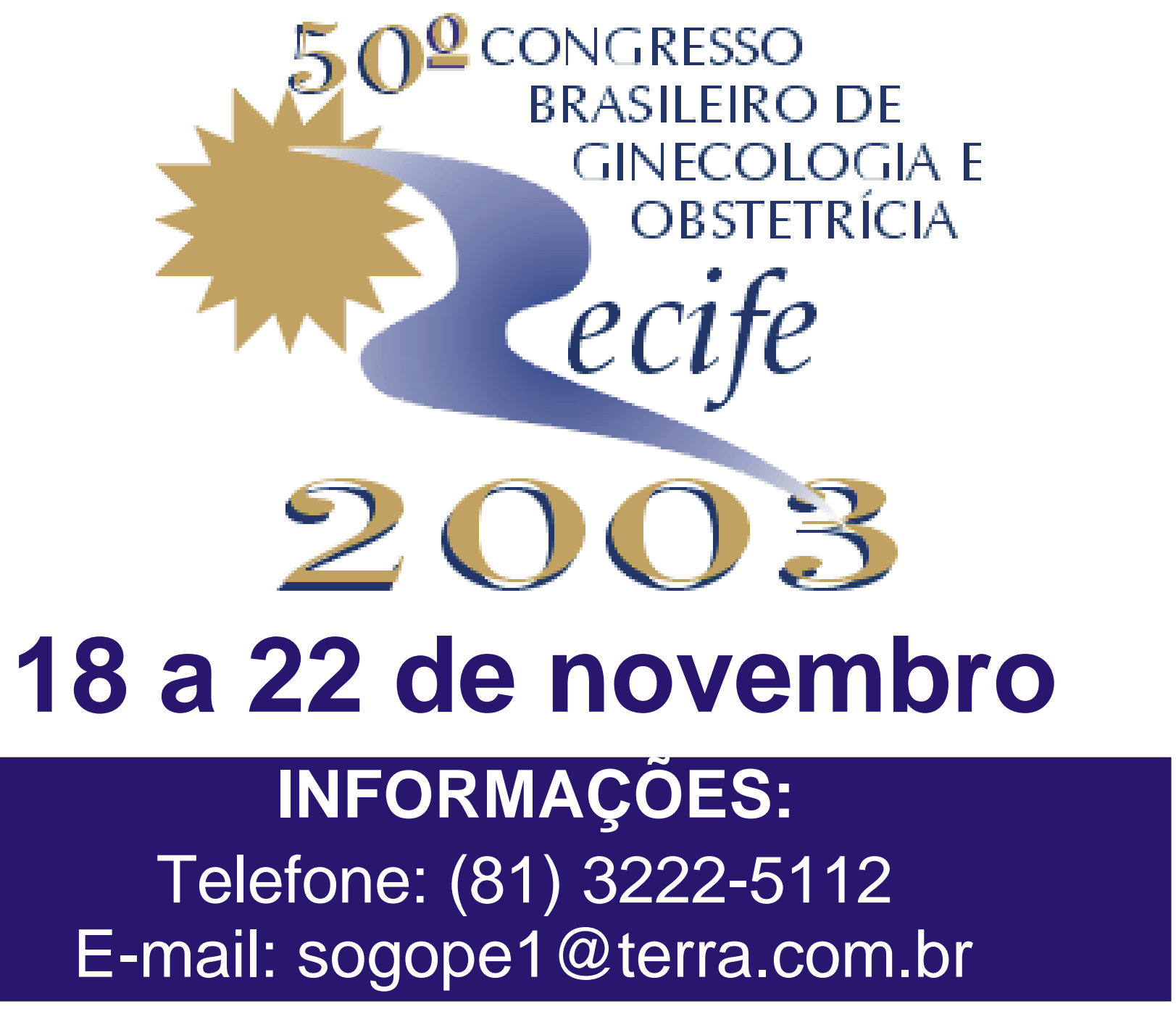

